\title{
THE GAUCHY PROBLEM FOR LINEAR PARTIAL DIFFERENTIAL EQUATIONS WITH RESTRICTED BOUNDARY CONDITIONS
}

\author{
E. P. Miles, JR. and ERNEST WILliams
}

We shall discuss solutions of linear partial differential equations of the form

$$
\Phi\left(D, x_{1}, x_{2}, \ldots x_{n}\right) u+\Psi(D, t) u=0,
$$

where $\Psi$ is an ordinary differential operator of order $s$ with respect to $t$. Our first theorem gives a solution of (1) for the Cauchy data;

$$
\begin{aligned}
u\left(x_{1}, x_{2}, \ldots x_{n}, 0\right) & =P\left(x_{1}, x_{2}, \ldots x_{n}\right), \\
\frac{\partial^{j} u}{\partial t^{j}}\left(x_{1}, x_{2}, \ldots x_{n}, 0\right) & =0, \quad j=1,2, \ldots, s-1,
\end{aligned}
$$

whenever the function $P$ is annihilated by a finite iteration of the operator $\Phi$. This situation occurs if $P$ is a polynomial and $\Phi$ any differential operator with constant coefficients and no constant term or if $P$ is polyharmonic and $\Phi$ the Laplacian operator. The solution hinges upon the integration of a finite system of ordinary differential equations.

THEOREM 1. Suppose for some integer $k$ we have

$$
\Phi^{k}(P) \neq 0, \Phi^{k+1}(P)=0 ;
$$

urther suppose that $u_{0}, u_{1}, \ldots u_{k}$ are a set of solutions of the system of ordinary differential equations

$$
\begin{aligned}
& \Psi\left(u_{j}\right)+u_{j-1}=0, \quad j=1,2, \ldots, k \\
& \Psi\left(u_{0}\right)=0,
\end{aligned}
$$

with initial conditions

$$
\begin{array}{cr}
u_{0}(0)=1, u_{j}(0)=0, & j \geqslant 1, \\
\frac{d^{m} u_{j}}{d t^{m}}(0)=0, & m=1,2, \ldots, s-1, \text { all } j ;
\end{array}
$$

then a solution of (1) satisfying (2) is

$$
u\left(x_{1}, x_{2}, \ldots x_{n}, t\right)=\sum_{j=0}^{k} \Phi^{j}(P) \cdot u_{j} .
$$

Received July 25, 1955. 
Proof.

$$
\begin{aligned}
{[\Phi+\Psi] u } & =[\Phi+\Psi] \sum_{j=0}^{k} \Phi^{j}(P) \cdot u_{j} \\
& =\sum_{j=0}^{k}[\Phi+\Psi] \Phi^{j}(P) \cdot u_{j} \\
& =P \cdot\left[\Psi\left(u_{0}\right)\right]+\sum_{j=1}^{k} \Phi^{j}(P)\left[\Psi\left(u_{j}\right)+u_{j-1}\right]+\Phi^{k+1}(P) \cdot u_{k} \\
& =0,
\end{aligned}
$$

by (3), (4) and the linearity of the operators $\Phi$ and $\Psi$. The conditions (5) on the $u_{j}$ ensure that (6) satisfies conditions (2) and the proof of the theorem is complete.

As an application of this theorem we construct a solution of

$$
a \frac{\partial u}{\partial x}+b \frac{\partial u}{\partial y}+c \frac{\partial u}{\partial t}=0, u(x, y, 0)=x^{2} y .
$$

We note that $\Phi(P)=2 a x y+b x^{2}, \Phi^{2}(P)=4 a b x+2 a^{2} y, \Phi^{3}(P)=6 a^{2} b$ and $\Phi^{4}(P)=0$.

$$
u_{0}=1, u_{1}=\frac{-t}{c}, u_{2}=\frac{t^{2}}{2 c^{2}}, u_{3}=\frac{-t^{3}}{6 c^{3}}
$$

and

$$
\text { (8) } \begin{aligned}
& u(x, y, t)=x^{2} y+\left(2 a x y+b x^{2}\right)\left(\frac{-t}{c}\right)+\left(4 a b x+2 a^{2} y\right)\left(\frac{t^{2}}{2 c^{2}}\right)+ \\
& 6 a^{2} b\left(\frac{-t^{3}}{6 c^{3}}\right) \\
&=
\end{aligned}
$$

The last form of (8) may also be obtained from the general solution of (7), $F(c x-a t, c y-b t)$, by requiring that it reduce to $x^{2} y$ when $t=0$.

An alternate set of Cauchy data frequently encountered for (1) when $\Psi$ is a second order operator is

(9) $u\left(x_{1}, x_{2}, \ldots x_{n}, 0\right)=0, \quad u_{t}\left(x_{1}, x_{2}, \ldots x_{n}, 0\right)=Q\left(x_{1}, x_{2}, \ldots x_{n}\right)$.

For this case an analogous theorem holds:

Theorem 2. Suppose (3) holds and $V_{0}, V_{1}, \ldots V_{k}$ are the solutions of

$$
\Psi\left(V_{0}\right)=0, \quad \Psi\left(V_{j}\right)+V_{j-1}=0, \quad j=1,2, \ldots k,
$$

with initial conditions

$$
\begin{array}{cl}
V_{j}(0)=0, & j=0,1, \ldots k, \\
V^{\prime}{ }_{0}(0)=1, \quad V^{\prime}{ }_{j}(0)=0, & j=1,2, \ldots k ;
\end{array}
$$

then the Cauchy problem for (1) with boundary values (9) has a solution

$$
u=\sum_{j=0}^{k} \Phi^{j}(Q) V_{j}
$$


The authors have recently obtained basic sets of homogeneous polynomial solutions (1) for the Laplace and wave equations in $k$ variables. Although Theorems 1 and 2 were not discovered until after these basic sets were developed, they provide a natural way for deriving them. Let us consider the wave equation in three space variables and one time variable,

$$
\nabla^{2} u-\frac{\partial^{2} u}{\partial t^{2}}=0
$$

A general homogeneous polynomial $W$, of degree $n$ in $(x, y, z, t)$, has $\left(\begin{array}{c}n+3 \\ 3\end{array}\right)$ arbitrary coefficients. Requiring $W$ to be a solution of (13) gives $\left(\begin{array}{c}n+1 \\ 3\end{array}\right)$ independent $t^{1}$ conditions on these coefficients showing that

$$
\left(\begin{array}{c}
n+3 \\
3
\end{array}\right)-\left(\begin{array}{c}
n+1 \\
3
\end{array}\right)=(n+1)^{2}
$$

of them are independent. We may construct our basic set of $(n+1)^{2}$ homogeneous polynomial solutions of degree $n$ for (13) by first applying Theorem 1 to each of the $\left(\begin{array}{c}n+2 \\ 2\end{array}\right)$ monomials $P(x, y, z)$ of type $x^{a} y^{b} z^{c}, a+b+c=n$ and then applying Theorem 2 to each of the $\left(\begin{array}{c}n+1 \\ 2\end{array}\right)$ monomials $Q(x, y, z)$ of type $x^{\alpha} y^{\beta} z^{\gamma}, \alpha+\beta+\gamma=n-1$. The resulting homogeneous polynomial solutions of (13) are

$$
\left(\begin{array}{c}
n+2 \\
2
\end{array}\right)+\left(\begin{array}{c}
n+1 \\
2
\end{array}\right)=(n+1)^{2}
$$

in number. The polynomials generated by Theorem 1 contain only one term of degree less than 2 in $t$, the generator term $x^{a} y^{b} z^{c}$; likewise those generated by Theorem 2 contain only one term $x^{\alpha} y^{\beta} z^{\gamma} t$ of lowest degree in $t$. Thus our set of solutions is independent and, since it is correctly numbered, is a basic set of solutions.

The basic set constructed in the above manner may be represented as follows: for each set of non-negative integers $a, b, c, d, a+b+c+d=n$, $d \leqslant 1$

$$
\begin{aligned}
W_{a, b, c, d}(x, y, z, t) & =\sum_{j=0}^{\left[\frac{1}{2} n\right]} \nabla^{2 j}\left(x^{a} y^{b} z^{c}\right) \cdot \frac{t^{2 j+d}}{(2 j+d) !} \\
& =\sum \frac{a ! b ! c !\left[\frac{1}{2} D\right] x^{A} y^{B} z^{C} t^{D}}{\left(\frac{a-A}{2}\right) !\left(\frac{b-B}{2}\right) !\left(\frac{c-C}{2}\right) ! A ! B ! C ! D !}
\end{aligned}
$$

Independence of these conditions may be readily established by a generalization of Whittaker's footnote on the corresponding harmonic polynomials (Whittaker \& Watson, Modern Analysis, 4th ed., p. 389) or, as suggested by the referee, by using an argument like that in Courant-Hilbert, vol. I, English ed., at the bottom of p. 512 . 
where the final summation extends over all non-negative $A, B, C$ and $D$ such that $A+B+C+D=n, A \equiv a, B \equiv b, C \equiv c, D \equiv d \bmod 2$, and $A \leqslant a$, $B \leqslant b, C \leqslant c$. The final form of (14) corresponds, except for a constant factor, to the basic set for the wave equation developed in (1), but the intermediate form given here for the first time is much simpler to describe and use.

Instead of trying to solve Cauchy's problem for the wave equation in terms of the basic set of polynomials (14) when our initial conditions are suitable we may use:

THEOREM 3. The solution of (13) with initial conditions

$$
u(x, y, z, 0)=P(x, y, z), \quad u_{t}(x, y, z, 0,)=Q(x, y, z),
$$

where $P(x, y, z)$ and $Q(x, y, z)$ are polyharmonic functions of order $p$ and $q$ respectively, is given by the sum:

$$
U(x, y, z, t)=\sum_{j=0}^{p-1} \nabla^{2 j}(P) \frac{t^{2 j}}{(2 j) !}+\sum_{k=0}^{q-1} \nabla^{2 k}(Q) \frac{t^{2 k+1}}{(2 k+1) !} .
$$

This theorem is established by applying Theorem 1 to (13) with boundary conditions, $u(x, y, z, 0)=P, u_{t}(x, y, z, 0)=0$ and Theorem 2 to (13) with boundary conditions, $u(x, y, z, 0)=0, u_{t}(x, y, z, 0)=Q$, and adding the resulting solutions.

As an illustration of Theorem 3 we display a solution of the wave equation (13), with initial conditions

(16) $u(x, y, z, 0)=x e^{x} \cos y \cdot z^{4}, \quad u_{t}(x, y, z, 0)=0$,

(17) $u(x, y, z, t)=e^{x} \cos y\left[x z^{4}+\left(z^{4}+6 x z^{2}\right) t^{2}+\left(2 z^{2}+x\right) t^{4}+\frac{1}{5} t^{6}\right]$.

In another recent paper (2) the authors gave basic sets of polynomial solutions for the Euler-Poisson-Darboux equation

$$
\nabla^{2} u-\left[u_{t t}+k t^{-1} u_{t}\right]=0
$$

and for the closely associated Beltrami equation. For the E.P.D. equation with $k>0$ a direct application of Theorem 1 to the generator monomials $x^{a} y^{b} z^{c}, a+b+c=n$, gives a more usable form of the basic sets similar to that given for the wave equation in (14) with $d=0$ and $t^{2 j} /(2 j)$ ! replaced by $t^{2 j} /(1+k)(3+k) \ldots(2 j-1+k) \cdot 2^{j} j !$

However, if $k$ is negative, the system of differential equations (4) associated with the solution of (18) under conditions (2) has a solution,

$$
\begin{aligned}
u_{j}=\sum_{n=0}^{j} \frac{a_{n} t^{2 j-2 n+1-k}}{2^{j-n}(j-n) !(1-k)(3-k) \ldots(2 j-2 n+1-k)} \\
\quad+\frac{t^{2 j}}{2^{j} j !(1+k)(3+k) \ldots(2 j-1+k)}
\end{aligned}
$$


provided $k \neq-1,-3, \ldots,-(2 j-1)$, which is not unique since the $a_{n}$ are arbitrary.

Weinstein has recently shown (see for instance (3)), that for odd negative integral values of $k$, solutions of (18) satisfying certain differentiability conditions exist only if the initial value function is polyharmonic of order $(1-k) / 2$ and that, in this case, the addition to the solution of any function of the type

$$
t^{1-k} u^{2-k}\left(x_{1}, \ldots x_{n}, t\right),
$$

$\left(u^{2-k}\right.$ denotes a solution of (18) with $k$ replaced by $\left.2-k\right)$ which vanishes with its $t$ derivative at $t=0$, gives another solution of the problem. We may illustrate this result of Weinstein by applying Theorem 1 to (18) with boundary conditions (16). As a first solution we may take $a_{n}=0, n=0,1,2, \ldots$ and obtain

$$
\text { (20) } \begin{aligned}
u(x, y, z, t) & =e^{x} \cos y\left[x z^{4}+\left(z^{4}+6 x z^{2}\right) \frac{t^{2}}{1+k}\right. \\
& \left.+\left(6 z^{2}+3 x\right) \frac{t^{4}}{(1+k)(3+k)}+\frac{3 t^{6}}{(1+k)(3+k)(5+k)}\right] .
\end{aligned}
$$

The solution (20) is invalid for $k=-1,-3,-5$, but holds for $k=-7$, $-9, \ldots$ etc. Since $x e^{x} \cos y \cdot z^{4}$ is polyharmonic of order $\frac{1}{2}\{1-(-7)\}=4$, this illustrates the result of Weinstein quoted above. As a further illustration of Weinstein's result we may let $a_{0} \neq 0$ in (19) and augment the solution (20) by

$$
\begin{aligned}
& a_{0} \frac{t^{1-k}}{1-k} e^{x} \cos y\left[x z^{4}+\left(z^{4}+6 x z^{2}\right) \frac{t^{2}}{[1+(2-k)]}\right. \\
& +\left(6 z^{2}+3 x\right) \frac{t^{4}}{[1+(2-k)][3+(2-k)]} \\
& \left.\quad+\frac{3 t^{6}}{[1+(2-k)][3+(2-k)][5+(2-k)]}\right]
\end{aligned}
$$

which is clearly an arbitrary constant multiple of $t^{1-k}$ by a solution of (18) with $k$ replaced by $(2-k)$ which vanishes with its $t$ derivative at $t=0$ for all negative $k$.

Where condition (3) does not hold, the above methods may lead us to solutions in infinite series form. As an illustration we may consider the vibrating string problem

$$
u_{x x}-a^{-2} u_{t t}=0, u(x, 0)=P(x), u_{t}(x, 0)=0, u(0, t)=u(L, t)=0,
$$

where the function $P(x)$ has the Fourier expansion

$$
P(x)=\sum_{1}^{\infty} b_{n} \sin \frac{n \pi x}{L} .
$$

We then obtain 


$$
\Phi^{j} P=\sum_{n=1}^{\infty}(-1)^{j} b_{n}\left(\frac{n \pi}{L}\right)^{2 j} \sin \frac{n \pi x}{L}
$$

and

$$
u_{j}=\frac{(a t)^{2 j}}{(2 j) !}
$$$$
j=0,1,2, \ldots
$$

Taking

$$
u=\sum_{j=0}^{\infty} \sum_{n=1}^{\infty}(-1)^{j} b_{n}\left(\frac{n \pi}{L}\right)^{2 j} \sin \frac{n \pi x}{L} \cdot \frac{(a t)^{2 j}}{(2 j) !}
$$

we have a formal solution. If the order of summation be interchanged we obtain

$$
u=\sum_{n=1}^{\infty} b_{n} \sin \frac{n \pi x}{L} \cos \frac{n \pi a t}{L},
$$

which is the usual form of the solution of this problem. The one dimensional heat flow problem may also be solved by this method but the applicability of the method to other problems, because of convergence questions, is a matter requiring further study.

\section{REFERENCES}

1. E. P. Miles, Jr. and Ernest Williams, $A$ basic set of homogeneous harmonic polynomials in $k$ variables, Proc. Amer. Math. Soc., 6 (1955), 191-194.

2. E. P. Miles, Jr. and Ernest Williams, A basic set of polynomial solutions for the EulerPoisson-Darboux and Beltrami equations. To appear in the American Mathematical Monthly.

3. Alexander Weinstein, On the wave equation and the equation of Euler-Poisson, Proc. Fifth Symposium in Applied Mathematics (New York, 1954), 137-147.

Alabama Polytechnic Institute, Auburn, Alabama 